https://doi.org/10.15407/ujpe64.8.725

J. PROCHÁZKA, V. KUNDRÁT, M.V. LOKAJÍČEK

The Czech Academy of Sciences, Institute of Physics

(18221 Prague 8,Czech Republic; e-mail: jiri.prochazka@fzu.cz,kundrat@fzu.cz, lokaj@fzu.cz)

\title{
MODELS OF ELASTIC pp SCATTERING AT HIGH ENERGIES - POSSIBILITIES, LIMITATIONS, ASSUMPTIONS, AND OPEN QUESTIONS
}

\begin{abstract}
The simplest collision process, the elastic scattering of protons, has been measured at various energies and in a broad interval of scattering angles. Its theoretical description is, however, much more delicate, than it may seem at first glance. The widely used eikonal model allowed one to analyze the pp elastic scattering data at an ISR energy of $52.8 \mathrm{GeV}$ and the TOTEM data at a much higher LHC energy of $8 \mathrm{TeV}$. The results represent the most detailed elaborated impact parameter analysis of pp data which has ever been performed. They have helped to identify several deeper open questions and problems concerning all widely used theoretical frameworks used for the description of the elastic pp scattering. The problems should be further studied and solved to derive some important proton characteristics which may be obtained with the help of the elastic scattering.

Keywords: proton-proton collisions, elastic scattering of hadrons, eikonal model, Coulombhadronic interference, central or peripheral scattering, impact parameter, WY approach.
\end{abstract}

\section{Introduction}

The elastic differential cross-section $\mathrm{d} \sigma / \mathrm{d} t$ represents a basic experimental characteristic established in the elastic collisions of hadrons. If the influence of spins is not considered then the $t$ (four momentum transfer squared) dependence exhibits a very similar structure in all cases of elastic scattering of charged hadrons at contemporary high energies: there is a peak at very low values of $|t|$, followed by a (nearly) exponential region, and then there is a dip-bump or shoulder structure at even higher values of $|t|$ practically for all colliding hadrons [1].

The measured differential elastic cross-section of two charged hadrons (protons) is standardly described with the help of the complete elastic scattering amplitude $F^{\mathrm{C}+\mathrm{N}}(s, t)$ as

$$
\frac{\mathrm{d} \sigma}{\mathrm{d} t}=\frac{\pi}{s p^{2}}|F(s, t)|^{2} .
$$

Here, $s$ is the square of the total collision energy, and $p$ is the value of the momentum of one incident proton in the center-of-mass system. The Cou-

\section{(c) J. PROCHÁZKA, V. KUNDRÁT, M.V. LOKAJÍČEK, 2019}

ISSN 2071-0194. Ukr. J. Phys. 2019. Vol. 64, No. 8 lomb amplitude $F^{\mathrm{C}}(s, t)$ is widely assumed to be well-known from QED (except from electromagnetic form factors). However, the $t$-dependence of the elastic hadronic amplitude $F^{\mathrm{N}}(s, t)$ is yet not fully known. The elastic scattering of two protons is kinematically the simplest collision process, but its description is not satisfactory in many aspects.

The description of the Coulomb-hadronic interference proposed by West and Yennie (WY) [2] in 1968 was widely used for the analysis of experimental data in the era of the ISR. However, several problems and limitations in the given model were identified later. This approach is discussed in sect. 2. The description is not usable for a reliable data analysis. It has, however, negatively influenced many recent models of elastic hadronic scattering. To overcome these problems, another approach based on the eikonal model framework has been developed. The results of analysis of experimental data using the eikonal model (under different assumptions) are summarized in sect. 3. The list of deeper open questions and problems identified in all contemporary descriptions of the elastic scattering is presented in sect. 4. Concluding remarks may be found in sect. 5. This paper very briefly summarizes the results obtained and discussed in more details in $[3,4]$. 


\section{Approach of West and Yennie}

In 1968, West and Yennie [2] derived for the complete amplitude the following simplified formula:

$F_{\mathrm{WY}}^{\mathrm{C}+\mathrm{N}}(s, t)= \pm \frac{\alpha s}{t} G_{1}(t) G_{2}(t) \mathrm{e}^{\mathrm{i} \alpha \phi(s, t)}+$

$+\frac{\sigma^{\mathrm{tot}, \mathrm{N}}(s)}{4 \pi} p \sqrt{s}(\rho(s)+\mathrm{i}) \mathrm{e}^{B(s) t / 2}$,

where (see also Locher 1967 [5])

$\alpha \phi(s, t)=\mp \alpha\left[\ln \left(\frac{-B(s) t}{2}\right)+\gamma\right]$

Here, $\alpha=1 / 137.036$ is the fine structure constant, $\gamma=0.577215 \ldots$ is the Euler constant, $G_{1}(t)$ and $G_{2}(t)$ are the electric dipole form factors (being put into formula (2) by hand at the very end of the whole derivation for point-like particles). The quantity $\sigma^{\text {tot,N }}$ is the total cross-section given by the optical theorem:

$\sigma^{\mathrm{tot}, \mathrm{N}}(s)=\frac{4 \pi}{p \sqrt{s}} \operatorname{Im} F^{\mathrm{N}}(s, t=0)$.

The simplified formula (2) was used widely mainly in the era of the ISR for the determination (often very misleadingly called a measurement) of three free parameters: $\sigma^{\text {tot, } \mathrm{N}}$, quantity $\rho(t=0)$, and diffractive slope $B(t=0)$. However, in the derivation of Eq. (2), two very strong assumptions concerning the $t$-dependence of the elastic hadronic amplitude were assumed to be valid at all kinematically allowed values of $t$ :

1. $t$-independence of the phase of $F^{\mathrm{N}}(s, t)$, i.e., the quantity

$\rho(s, t)=\frac{\operatorname{Re} F^{\mathrm{N}}(s, t)}{\operatorname{Im} F^{\mathrm{N}}(s, t)}$

was assumed to be $t$-independent;

2. purely exponential $t$-dependence of $\left|F^{\mathrm{N}}(s, t)\right|$, i.e., the diffractive slope defined as

$B(s, t)=\frac{\mathrm{d}}{\mathrm{d} t}\left[\ln \frac{\mathrm{d} \sigma^{\mathrm{N}}}{\mathrm{d} t}(s, t)\right]=\frac{2}{\left|F^{\mathrm{N}}(s, t)\right|} \frac{\mathrm{d}}{\mathrm{d} t}\left|F^{\mathrm{N}}(s, t)\right|$

was assumed to be $t$-independent.

It has been shown in [6] that the first assumption must be valid otherwise the relative phase $\phi(s, t)$ becomes a complex function, which would lead to a contradiction (the relative phase has been defined as a real function [2]). The second assumption is in contradiction to the observed dip-bump structure in measured $\mathrm{d} \sigma / \mathrm{d} t$ data. Several other limitations and problems in the derivation of the simplified formula (2) or its application in the forward region were identified later, see $[3,7]$ for corresponding details and further references. The approach of WY is inapplicable for the reliable analysis of experimental data. Many recent models of elastic hadronic amplitude have been negatively influenced by the simplified formula (2). The models have been typically constrained by the values of $\sigma^{\text {tot,N }}$, quantity $\rho(t=0)$, and $B(t=0)$ determined on the basis of the simplified formula, even though they have corresponded to the strongly $t$-dependent quantities $B(t)$ and $\rho(t)$. The measured differential cross-section data have been, therefore, described inconsistently.

\section{Eikonal Model Approach}

\subsection{Theoretical background}

In order to avoid (some of) the discrepancies and limitations related to the simplified WY formula, another approach to the description of the Coulomb-hadronic interference based on the eikonal model was proposed in 1994 by Kundrát and Lokajíček [8]. This widely used theoretical framework allowed one to derive a more general formula for the complete elastic scattering amplitude valid for any $t$-dependence of the phase and modulus of $F^{\mathrm{N}}(s, t)$ at a given (high) collision energy $\sqrt{s}$ and any value of $t$ :

$F^{\mathrm{C}+\mathrm{N}}(s, t)= \pm \frac{\alpha s}{t} G_{\mathrm{eff}}^{2}(t)+F^{\mathrm{N}}(s, t)[1 \mp \mathrm{i} \alpha \bar{G}(s, t)]$,

where

$\bar{G}(s, t)=\int_{t_{\min }}^{0} \mathrm{~d} t^{\prime}\left\{\ln \left(\frac{t^{\prime}}{t}\right) \frac{\mathrm{d}}{\mathrm{d} t^{\prime}}\left[G_{\mathrm{eff}}^{2}\left(t^{\prime}\right)\right]-\right.$

$\left.-\frac{1}{2 \pi}\left[\frac{F^{\mathrm{N}}\left(s, t^{\prime}\right)}{F^{\mathrm{N}}(s, t)}-1\right] I\left(t, t^{\prime}\right)\right\}$

and

$I\left(t, t^{\prime}\right)=\int_{0}^{2 \pi} \mathrm{d} \Phi^{\prime \prime} \frac{G_{\text {eff }}^{2}\left(t^{\prime \prime}\right)}{t^{\prime \prime}}$

here, $t^{\prime \prime}=t+t^{\prime}+2 \sqrt{t t^{\prime}} \cos \Phi^{\prime \prime}$. The upper (lower) sign corresponds to the scattering of particles with the same (opposite) charges. $G_{\text {eff }}^{2}$ is the effective form 
factor squared reflecting the electromagnetic structure of colliding protons and was introduced in [9] as

$G_{\mathrm{eff}}^{2}(t)=\frac{1}{1+\tau}\left[G_{\mathrm{E}}^{2}(t)+\tau G_{\mathrm{M}}^{2}(t)\right], \quad \tau=-\frac{t}{4 m^{2}}$,

where $G_{\mathrm{E}}$ and $G_{\mathrm{M}}$ stand for the electric and magnetic form factors, and $m$ is the proton mass. The interference formula given by Eq. (7) allows one to study the $t$-dependence of the elastic hadronic amplitude and corresponding $b$-dependent properties consistently in the whole measured $t$ range.

The $b$-dependent characteristics of $p p$ collisions are standardly analyzed with the help of the FourierBessel transform. It should be, however, consistent with a finite allowed region of the variable $t$ and finite collision energies [10] (which is often not respected at all)

$$
\begin{aligned}
& h_{\mathrm{el}}(s, b)=h_{1}(s, b)+h_{2}(s, b)= \\
& =\frac{1}{4 p \sqrt{s}} \int_{-\infty}^{t_{\min }} F^{\mathrm{N}}(s, t) J_{0}(b \sqrt{-t}) \mathrm{d} t+ \\
& +\frac{1}{4 p \sqrt{s}} \int_{t_{\mathrm{min}}}^{0} F^{\mathrm{N}}(s, t) J_{0}(b \sqrt{-t}) \mathrm{d} t .
\end{aligned}
$$

In this case, the unitarity equation in the $b$-space is

$\operatorname{Im} h_{1}(s, b)=\left|h_{1}(s, b)\right|^{2}+g_{1}(s, b)+K(s, b)$.

Here, $g_{1}(s, b)$ is a real inelastic overlap function which has been introduced in a similar way as the complex elastic amplitude in Eq. (11). The complex function $h_{1}(s, b)$ and real functions $g_{1}(s, b)$ oscillate at finite energies. The oscillations can be removed, if a real function $c(s, b)=-\operatorname{Im} h_{2}(s, b)$ fulfilling some mathematical conditions is added to both sides of the unitarity equation (12) [3]. It is then possible to define, at finite energies, the total, elastic, and inelastic profile functions $D^{\mathrm{X}}(s, b)(\mathrm{X}=\mathrm{tot}, \mathrm{el}$, inel)

$$
\begin{aligned}
& D^{\text {el }}(s, b) \equiv 4\left|h_{1}(s, b)\right|^{2}, \\
& D^{\text {tot }}(s, b) \equiv 4\left(\operatorname{Im} h_{1}(s, b)+c(s, b)\right), \\
& D^{\text {inel }}(s, b) \equiv 4\left(g_{1}(s, b)+K(s, b)+c(s, b)\right)
\end{aligned}
$$

and rewrite the unitarity condition in the $b$-space as

$D^{\text {tot }}(s, b)=D^{\text {el }}(s, b)+D^{\text {inel }}(s, b)$.
These profile functions (sometimes called overlap functions) represent main $b$-dependent characteristics. They are used to define the root-mean-squared impact parameter $\sqrt{\left\langle b^{2}\right\rangle^{\mathrm{X}}}$ corresponding to the total, elastic, or inelastic hadron collisions.

Nearly all contemporary models of elastic hadron scattering a priori strongly constrain the elastic hadronic amplitude $F^{\mathrm{N}}(s, t)$ from the very beginning without sufficient reasoning, by requiring

1. dominance of the imaginary part of $F^{\mathrm{N}}(s, t)$ in a quite broad interval of $t$ in the forward region close to $t=0$;

2. vanishing of the imaginary part of $F^{\mathrm{N}}(s, t)$ at (or around) the $\operatorname{dip} t=t_{\operatorname{dip}}$ (wrongly reasoned as a consequence of the minimum of $\mathrm{d} \sigma / \mathrm{d} t$ at $\left.t_{\mathrm{dip}}\right)$;

3.values of $\left.\sigma^{\text {tot,N }}\right], B(t=0)$ and $\rho(t=0$ ) (often misleadingly denoted as "measurement") obtained from the simplified WY formula;

4. change of a sign of the real part of $F^{\mathrm{N}}(s, t)$ at "low" values of $|t|$ (motivated by Martin's theorem [11] derived under certain (asymptotic) conditions).

The corresponding $t$-dependence of $F^{\mathrm{N}}(s, t)$ (its phase) is strongly constrained by these requirements. It may be shown that mainly the first requirement leads to the central behavior of elastic collisions corresponding to $\sqrt{\left\langle b^{2}\right\rangle^{\mathrm{el}}}<\sqrt{\left\langle b^{2}\right\rangle^{\mathrm{inel}}}$. The structure of protons which would correspond to this behavior has never been sufficiently explained.

One may, therefore, ask if it is possible to obtain a description of data which would lead to the peripheral behavior of elastic collisions $\sqrt{\left\langle b^{2}\right\rangle^{\mathrm{el}}}>\sqrt{\left\langle b^{2}\right\rangle^{\text {inel }}}$ (without imposing the unreasoned constrains above). It was shown in 1981 [12] that the peripheral solution of the scattering problem may be obtained, if the hadronic phase has specific $t$-dependence.

\subsection{Analysis of Measured Data}

One may try to determine $F^{\mathrm{N}}(s, t)$ on the basis of experimental data under a given set of assumptions (constraints) and to study their impact on values of determined hadronic quantities. The eikonal interference formula given by Eqs. (7) to (9) may be used to subtract the Coulomb effect from the measured elastic pp $\mathrm{d} \sigma / \mathrm{d} t$ data at a given energy. The analysis of experimental elastic data in the full measured region of $t$ values with the help of Eqs. (7) to (9) (with either effective electric or effective electromagnetic proton form factors determined from the ep scattering) 
Comparison of several hadronic quantities characterizing the pp elastic scattering at energies of $52.8 \mathrm{GeV}$ and $8 \mathrm{TeV}$

\begin{tabular}{|c|c|c|c|c|}
\hline $\begin{array}{l}\text { Particle types } \\
\sqrt{s}[\mathrm{GeV}] \\
\text { Fit } \\
\text { Case }\end{array}$ & $\begin{array}{c}\text { pp } \\
52.8 \\
1 \\
\text { central }\end{array}$ & $\begin{array}{c}\mathrm{pp} \\
52.8 \\
2 \\
\text { peripheral }\end{array}$ & $\begin{array}{c}\text { pp } \\
8000 \\
1 \\
\text { central }\end{array}$ & $\begin{array}{c}\text { pp } \\
8000 \\
2 \\
\text { peripheral }\end{array}$ \\
\hline $\begin{array}{l}\rho(t=0) \\
B(t=0)\left[\mathrm{GeV}^{-2}\right] \\
\sigma^{\text {tot,N }}[\mathrm{mb}] \\
\sigma^{\mathrm{el}, \mathrm{N}}[\mathrm{mb}] \\
\sigma^{\mathrm{inel}}[\mathrm{mb}] \\
\sigma^{\mathrm{el}, \mathrm{N}} / \sigma^{\mathrm{tot}, \mathrm{N}} \\
\mathrm{d} \sigma^{\mathrm{N}} / \mathrm{d} t(t=0)\left[\mathrm{mb} . \mathrm{GeV}^{-2}\right]\end{array}$ & $\begin{array}{c}0.0763 \pm 0.0017 \\
13.515 \pm 0.035 \\
42.694 \pm 0.033 \\
7.469 \\
35.22 \\
0.1750 \\
93.67\end{array}$ & $\begin{array}{c}0.0827 \pm 0.0016 \\
13.444 \pm 0.036 \\
42.861 \pm 0.034 \\
7.539 \\
35.32 \\
0.1759 \\
94.51\end{array}$ & $\begin{array}{c}0.122 \pm 0.018 \\
21.021 \pm 0.085 \\
103.44 \pm 0.35 \\
27.6 \\
75.9 \\
0.267 \\
555\end{array}$ & $\begin{array}{c}0.149 \pm 0.016 \\
20.829 \pm 0.055 \\
104.12 \pm 0.31 \\
28.0 \\
76.1 \\
0.269 \\
566\end{array}$ \\
\hline $\begin{array}{l}\sqrt{\left\langle b^{2}\right\rangle^{\text {tot }}}[\mathrm{fm}] \\
\sqrt{\left\langle b^{2}\right\rangle^{\mathrm{el}}}[\mathrm{fm}] \\
\sqrt{\left\langle b^{2}\right\rangle^{\text {inel }}}[\mathrm{fm}] \\
D^{\text {tot }}(b=0) \\
D^{\mathrm{el}}(b=0) \\
D^{\text {inel }}(b=0)\end{array}$ & $\begin{array}{c}1.026 \\
0.6778 \\
1.085 \\
1.29 \\
0.530 \\
0.762\end{array}$ & $\begin{array}{c}1.023 \\
1.959 \\
0.671 \\
1.30 \\
0.0342 \\
1.27\end{array}$ & $\begin{array}{c}1.39 \\
2.01 \\
0.980 \\
1.03\end{array}$ & $\begin{array}{c}0.970 \\
2.04 \\
0.205 \\
1.84\end{array}$ \\
\hline
\end{tabular}

requires a convenient parametrization of the complex elastic hadronic amplitude, i.e., of its modulus and phase:

$F^{\mathrm{N}}(s, t)=\mathrm{i}\left|F^{\mathrm{N}}(s, t)\right| \mathrm{e}^{-\mathrm{i} \zeta^{\mathrm{N}}(s, t)}$.

The modulus can be parametrized as

$$
\begin{aligned}
& \left|F^{\mathrm{N}}(s, t)\right|=\left(a_{1}+a_{2} t\right) \mathrm{e}^{b_{1} t+b_{2} t^{2}+b_{3} t^{3}}+ \\
& +\left(c_{1}+c_{2} t\right) \mathrm{e}^{d_{1} t+d_{2} t^{2}+d_{3} t^{3}},
\end{aligned}
$$

and the phase can be parametrized as

$\zeta^{\mathrm{N}}(s, t)=\zeta_{0}+\zeta_{1}\left|\frac{t}{t_{0}}\right|^{\kappa} \mathrm{e}^{\nu t}, \quad t_{0}=1 \mathrm{GeV}^{2}$.

This parametrization of the phase allows very different $t$-dependences according to the values of free parameters. It allows a rather fast increase of $\zeta^{\mathrm{N}}(s, t)$ with $|t|$, which is inevitable for increasing the value of $\sqrt{\left\langle b^{2}\right\rangle^{\mathrm{el}}}$ (for details, see, e.g., [3, $\left.7,8,12,13\right]$ ). All parameters specifying the modulus and phase of the elastic hadronic amplitude $F^{\mathrm{N}}(s, t)$ may be energydependent. The parameter $\kappa$ needs to be chosen as a positive integer to keep the analyticity of $F^{\mathrm{N}}(s, t)$.

Many fits of measured differential cross-section at $52.8 \mathrm{GeV}$ [14] and $8 \mathrm{TeV}$ data [15] under different additional constraints have been recently performed in [3] (see also [7]). Table shows two fits at each energy. Fit 1 corresponds to the widely imposed requirements on $F^{\mathrm{N}}(s, t)$ in many models of elastic scattering discussed in sect. 3.1. This leads to the central behavior of elastic collisions. Fit 2 corresponds to the peripheral picture of elastic collisions, and it has been obtained without imposing the strong and unreasoned constraints. The $b$-dependent profile functions given by Eqs. (13) to (15) corresponding to Fit 1 (central) and Fit 2 (peripheral) at an energy of $52.8 \mathrm{GeV}$ are plotted in Figure.

The impact of a choice of the form factor (effective electric or effective electromagnetic one) has been found to be negligible or very small. The $t$ dependence of the hadronic phase $\zeta^{\mathrm{N}}(s, t)$ has, however, a fundamental impact on the character of collisions in the $b$-space. In a central case, relation $\sqrt{\left\langle b^{2}\right\rangle^{\mathrm{el}}}<\sqrt{\left\langle b^{2}\right\rangle^{\text {tot }}}$ holds. But, in the peripheral alternative, the relation is reversed. It may be also interesting to note that Martin's theorem [11] is fulfilled in the central, as well as peripheral, alternative (at both energies).

\section{Open Questions and Problems}

We have reviewed many (all widely discussed) historical and contemporary models concerning the de-

ISSN 2071-0194. Ukr. J. Phys. 2019. Vol. 64, No. 8 


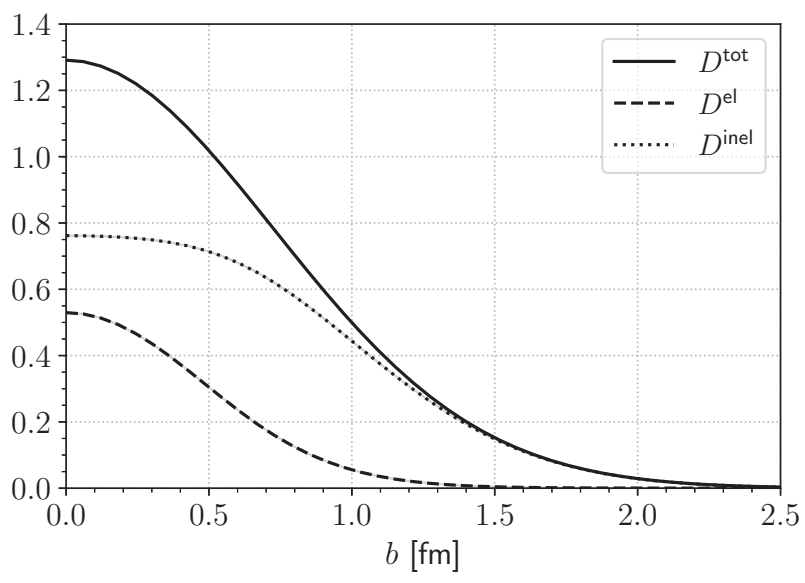

(a) central case - Fit 1

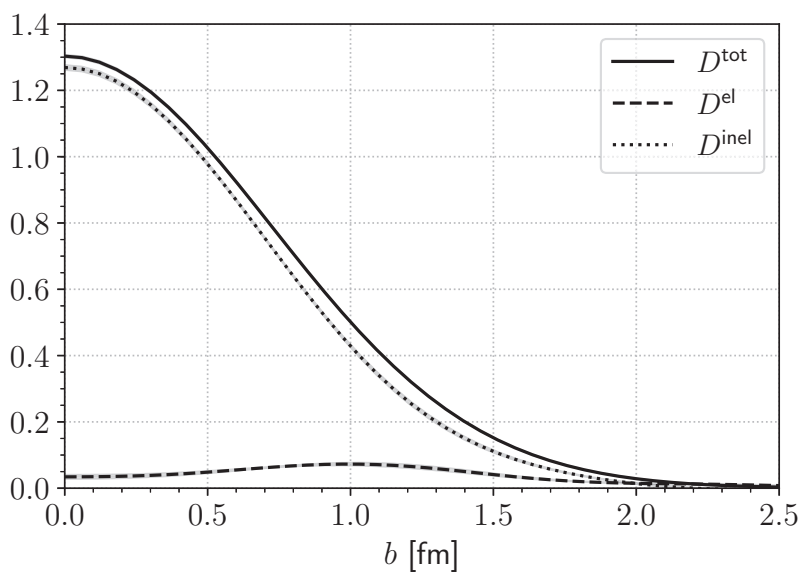

(b) peripheral case - Fit 2

Proton-proton profile functions $D(b)$ at an energy of $52.8 \mathrm{GeV}$. Full line corresponds to the total profile function, dashed line to the elastic one, and dotted line to the inelastic one

scription of elastic collisions and performed various fits of data under different conditions in order to better understand the processes with strongly interacting particles. On the basis of these studies, we have identified some deeper problems and open questions in all models and theoretical frameworks used in the description of the elastic scattering:

1. Coulomb interaction and experimental conditions;

a) (non-)divergence at $t=0$

b) multiple collisions

c) electromagnetic form factors

2. Different mechanisms of Coulomb and strong forces;

3. Different types of short-ranged (contact) interactions;

4. Properties of the $S$ matrix and the structure of a Hilbert space;

5. Optical theorem;

6. Determination of the $b$-dependent probability functions of hadron collisions;

7. Distribution of elastic scattering angles for a given value of the impact parameter;

8. Increase in the integrated total, elastic, and inelastic cross-sections and the dimensions of colliding particles in dependence on the collision energy;

9. extrapolations outside measured regions.

The identified open problems 1-7 were published in [4]. One may find there also the historical context concerning the dependence of proton collisions on the impact parameter, which is not widely known. Prob- lems related specifically to the derivation of the optical theorem in particle physics are discussed in [16]. Open questions 8 and 9 are discussed in [3].

\section{Conclusion}

The simplified WY formula given by Eq. (2) and (3) was used widely in the era of the ISR for the analysis of experimental data. Determined values of $\left.\sigma^{\mathrm{tot}, \mathrm{N}}\right]$, $B(t=0)$, and $\rho(t=0)$ (at a given collision energy) on the basis of this model have often been denoted misleadingly as "measurement". Many problems and limitations in the derivation of the formula, as well as in its application to data, have been identified, see sect. 2. The WY approach should be, therefore, abandoned in the era of the LHC, as it may lead to wrong physical conclusions. It should not be used for constraining the hadronic models based on assumptions inconsistent with the assumptions used in the derivation of a simplified WY model. One should look for the other description of the elastic scattering of (charged) hadrons.

The eikonal model approach is more general and relevant for the analysis of elastic scattering data at the present time, than the (over)simplified WY model. The former allows one to study the $t$ dependence of the elastic hadronic amplitude and corresponding hadronic quantities. It is more fundamental than the other contemporary models of elastic scattering as it may be used for the description of the Coulomb-hadronic interference and to consider the dependence of collisions on the impact parameter (in 
order not to mix collisions corresponding to different values of the impact parameter). We have analyzed elastic scattering data at $52.8 \mathrm{GeV}$ and $8 \mathrm{TeV}$ with the help of the eikonal model under different assumptions consistently in the whole measured $t$-range to see the impact on values of different physical quantities, see sect. 3 .

This analysis of elastic scattering data with the use of the eikonal model approach has been prepared for the analysis of TOTEM data at the LHC. The first measurement of elastic differential pp data at the LHC energy of $8 \mathrm{TeV}$ in the Coulomb-hadronic region published by TOTEM [15] contains the first analysis of the $8 \mathrm{TeV}$ data using the eikonal model approach.

The results of our analysis (see sect. 3 and $[3,7]$ for more details and further references) represent the most elaborated impact parameter analysis of elastic pp collision data which has ever been performed. On the basis of our results, it may be concluded that the transparency of protons during elastic collisions (derived in widely used models of elastic pp scattering) has been based on unreasoned and unnecessary assumptions; the corresponding structure of protons has never been sufficiently explained in the literature. It is possible to say that there is no argument against the more realistic interpretation of elastic processes being peripheral and the protons regarded as rather compact (non-transparent) objects during elastic collisions.

We have reviewed basically all publicly available descriptions (models) of elastic hadron scattering over many years. Several deeper problems and open questions in all contemporary theoretical approaches (this includes WY model, eikonal model, Regge-based approaches, QCD-inspired approaches, ...) have been identified, see sect. 4. The proper analysis of hadron collisions in dependence on the impact parameter may provide an important insight concerning the shapes and dimensions (and other properties) of colliding particles, which can be hardly obtained in a different way. However, one should carefully study the assumptions involved in any collision model and test the consequences. It is also necessary to solve all the known fundamental problems and open questions in any contemporary description of the elastic pp scattering before making the far-reaching conclusions concerning the structure and properties of collided particles.
Further comments and new ideas how to move forward may be found in $[4,17]$. The more fundamental analysis of the whole contemporary state of fundamental physical researches has been recently summarized in [18]. It has been argued that, to make progress in physics, one needs to return to causal ontology and falsification approach (i.e., the logic and systematic analysis of involved assumptions). In our opinion, our results may be important for new trends not only in high-energy physics, but in physics in general.

We would like to thank to the organizers, especially to L. Jenkovszky, of the "New Trends in High-Energy Physics" conference which took place in Odessa (Ukraine) in May 12-18 (2019) for the opportunity to present and discuss the achieved results.

1. M.K. Carter, P.D.B. Collins, M.R. Whalley. Compilation of nucleon-nucleon and nucleon-antinucleon elastic scattering data. Preprint RAL-86-002 (1986).

2. G.B. West, D.R. Yennie. Coulomb interference in highenergy scattering. Phys. Rev. 172, 1413 (1968).

3. J. Procházka, V. Kundrát. Eikonal model analysis of elastic proton-proton collisions at $52.8 \mathrm{GeV}$ and $8 \mathrm{TeV}$. arXiv:1606.09479 (2019).

4. J. Procházka, M. V. Lokajíček, V. Kundrát. Dependence of elastic hadron collisions on impact parameter. Eur. Phys. J. Plus 131, 147 (2016).

5. M.P. Locher. Relativistic treatment of structure in the Coulomb interference problem. Nucl. Phys. B 2, 525 (1967).

6. V. Kundrát, M. Lokajíček, I. Vrkoč. Limited validity of West and Yennie interference formula for elastic scattering of hadrons. Phys. Lett. B 656, 182 (2007).

7. J. Procházka. Elastic Proton-Proton Collisions at High Energies, PhD thesis (Charles University, 2018).

8. V. Kundrát, M. Lokajíček. High-energy scattering amplitude of unpolarized and charged hadrons. Z. Phys. C 63, 619 (1994).

9. M.M. Block. Are we really measuring the phase of the nuclear scattering amplitude? Phys. Rev. D 54, 4337 (1996).

10. M.M. Islam. Lectures in theoretical Physics. Edited by A.O. Barut and W.E. Brittin (Gordon and Breach, 1968), Vol. 10, B, 97.

11. A. Martin. A theorem on the real part of the highenergy scattering amplitude near the forward direction. Phys. Lett. B 404, 137 (1997).

12. V. Kundrát, M. Lokajíček, jr., M. Lokajíček. Can quantummechanical description of physical reality be considered complete? Czech. J. Phys. B 31, 1334 (1981).

ISSN 2071-0194. Ukr. J. Phys. 2019. Vol. 64, No. 8 
13. V. Kundrát, M. Lokajíček, D. Krupa. Impact parameter structure derived from elastic collisions. Phys. Lett. B 544, 132 (2002).

14. J. Bystricky et al. Nucleon-Nucleon and Kaon-Nucleon Scattering. In Landolt-Bornstein, Numerical Data and Functional Relationships in Science and Technology, New Series. Edited by H. Schopper (Springer, 1980), Vol. 9.

15. TOTEM Collaboration. Measurement of elastic pp scattering at $\sqrt{s}=8 \mathrm{TeV}$ in the Coulomb-nuclear interference region - determination of the $\rho$-parameter and the total cross-section. Eur. Phys. J. C 76, 661 (2016).

16. J. Procházka, V. Kundrát, M.V. Lokajíček. Elastic scattering of hadrons without optical theorem. arXiv:1502.00468 (2015).

17. M. V. Lokajíček, V. Kundrát, J. Procházka. Schrödinger equation and (future) quantum physics. In Advances in Quantum Mechanics, Edited by Paul Bracken (Intech, 2013).
18. M. V. Lokajíček, J. Procházka. The Contemporary State of Fundamental Physical Research and the Future Path to Scientific Knowledge. arXiv:1610.08331 (2019).

Received 08.07.19

Ї. Прохазка, В. Кундрат, М.В. Локаӥчек

МОДЕЛІ ПРУЖНОГО рр-РОЗСІЯННЯ -

МОЖЛИВОСТІ, ОБМЕЖЕННЯ ТА ПИТАННЯ

$\mathrm{P}$ е $з$ ю м е

Найпростіший процес зіткнень, а саме пружне розсіяння протонів вимірювалось при різних енергіях та широкому інтервалі кутів розсіяння. Відповідний теоретичний опис, однак, набагато делікатніший, ніж може здаватися. Широко відома ейкональна модель дозволила провести аналіз пружних рр-даних при енергіях прискорювачів ISR, 52,8 ГеВ та LHC 8 TeB. Наші результати представляють найдетальніший та ретельно опрацьований прицільний аналіз рр-даних. Вони допомогли прояснити ряд питань та проблем опису пружного розсіяння протонів. Цю програму потрібно продовжити. 